\title{
Asymmetric catalytic addition of diorganozinc reagents to imines: Scope and application*
}

\author{
André B. Charette $\ddagger$, Alessandro A. Boezio, Alexandre Côté, \\ Elaine Moreau, Julien Pytkowicz, Jean-Nicolas Desrosiers, and \\ Claude Legault
}

Département de Chimie, Université de Montréal, Montréal H3C 3J7, Canada

\begin{abstract}
The copper-catalyzed diorganozinc addition to $\mathrm{N}$-diphenylphosphinoylimines was shown to proceed with a very high degree of enantiocontrol if the reaction was run in the presence of Me-DuPHOS monoxide ligand (BozPHOS). The scope of the reaction is described as well as our efforts to identify the nature of the enantioactive metal complex responsible for the high asymmetric induction.
\end{abstract}

Keywords: asymmetry; enantioselectivity; catalysis; copper; redox; sulfinate; diorganozinc; BozPHOS; $\alpha$-chiral amines; phosphinoylimine.

The asymmetric catalytic addition of nucleophiles to imines is a potentially very important process [1] owing to the predominant role that $\alpha$-chiral amines play in biologically important systems. Although these subunits are widely found in pharmaceutically important compounds and are the key component of unnatural amino acids, it is only recently that methods based on asymmetric catalysis involving nucleophilic addition of organometallic reagents to imines have appeared [2]. Traditionally, these chiral building blocks have been prepared either using resolution techniques or chiral auxiliary-based approaches (Fig. 1).

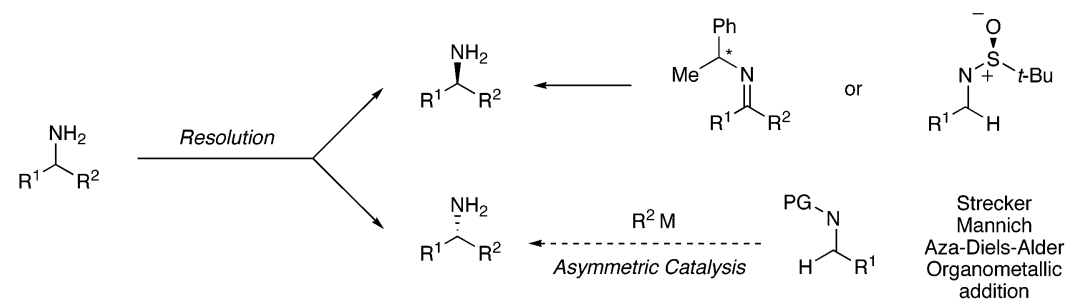

Fig. 1

Among the different methods that are available for the asymmetric catalytic addition of dialkylzinc reagents to imines, those reported by Tomioka [3], Hoveyda/Snapper [4], and Bräse [5] stand out as the most effective ones so far (eqs. 1-3).

\footnotetext{
*Pure Appl. Chem. 77, 1087-1296. An issue of reviews and research papers based on lectures presented at the $15^{\text {th }}$ International Conference on Organic Synthesis (ICOS-15), held in Nagoya, Japan, 1-6 August 2004, on the theme of organic synthesis.

¥Corresponding author
} 


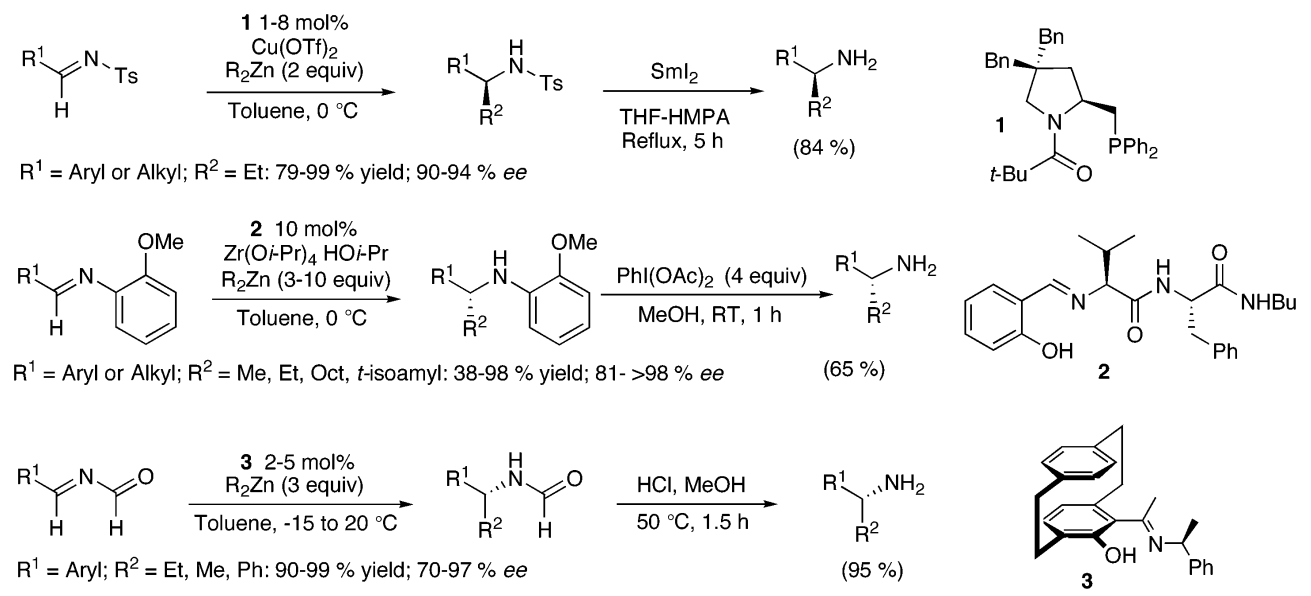

Two additional catalytic systems have been reported for the addition of diethylzinc to $N$-tosylimines [6,7]. Wang and Shi have shown that a binaphthyldiamine derivative $\mathbf{4}$ catalyzes the addition of diethylzinc to imines (eq. 4).

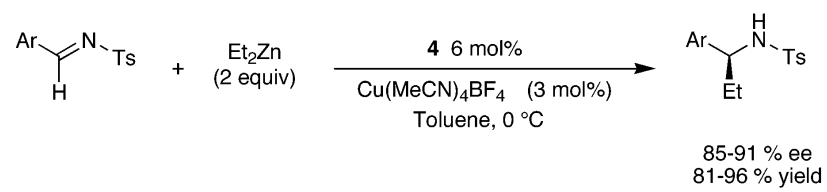<smiles>CCNc1ccc2ccccc2c1-c1c(N=CP)ccc2ccccc12</smiles>

Our interest in developing new methods for the synthesis of chiral amines led us to investigate whether it would be possible to generate an alternative approach from the highly electrophilic $N$-phosphinoylimines. Two advantages of this method would be the ease of cleavage of the nitrogen protecting group and good electrophilicity [8,9]. However, developing an asymmetric catalytic version is extremely challenging since most systems that have been developed with this electrophile so far for the addition of diorganozinc reagents involve a stoichiometric amount of a chiral controller [10].

Recently, we have disclosed that Me-DuPHOS/copper(II) triflate was a very effective chiral catalyst for the addition of diorganozinc reagents to $N$-phosphinoylimines (eq. 5) [11].

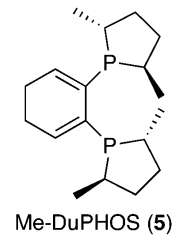

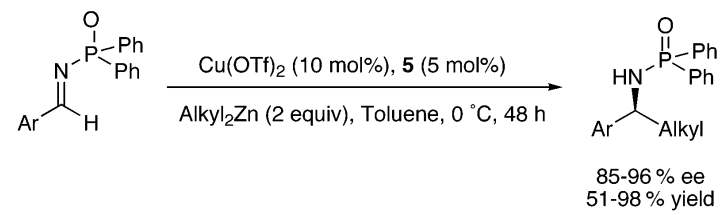

This method is quite effective for the addition of diethylzinc and higher diorganozinc reagents to imines producing the protected $\alpha$-chiral amine in high enantiomeric excess. However, the efficient addition of dimethylzinc is problematic. In fact, other catalytic systems use up to 10 equiv of dimethylzinc, but still obtain relatively low yields (51\%). This is a common feature between the Me-DuPHOS system and all of the other most successful systems to date in which a large excess of dimethylzinc is required and modest enantiomeric excesses (84-88 \%) and/or conversions are observed. Using the copper Me-DuPHOS system, very low conversions and enantioselectivities were observed if the number of equivalents was reduced to a practical level. The lack of reactivity of $\mathrm{Me}_{2} \mathrm{Zn}$ combined with the importance of the addition products in synthesis convinced us that new and more effective chiral ligands should be developed for this transformation. After considerable ligand screening, we were pleased to find that the bis(phosphine) monoxide derived from Me-DuPHOS produced spectacular results. 
The rationale for the ligand modification was triggered by the observation that a significant rate of acceleration for this reaction was observed with monodentate phosphines as well as with bidentate ligands bearing one labile group. Furthermore, we observed that the reaction was completely suppressed if $\geq 2$ equiv of Me-DuPHOS relative to $\mathrm{Cu}$ were used. These results led us to screen a large number of bis(phosphine) monoxide ligands [12,13], and we were quite pleased to discover that Me-DuPHOS monoxide (BozPHOS, 6) was a very effective ligand for the copper-catalyzed diorganozinc addition to $\mathrm{N}$-phosphinoylimines. This ligand is easily prepared in 3 steps and $90 \%$ overall yield from Me-DuPHOS (Scheme 1). Monoprotection of the Me-DuPHOS as its phosphine-borane complex was very effective even if more than 1 equiv of borane was used since we were unable to generate the bis(phosphine borane) complex. A subsequent oxidation with hydrogen peroxide followed by deprotection with DABCO led to BozPHOS (6).
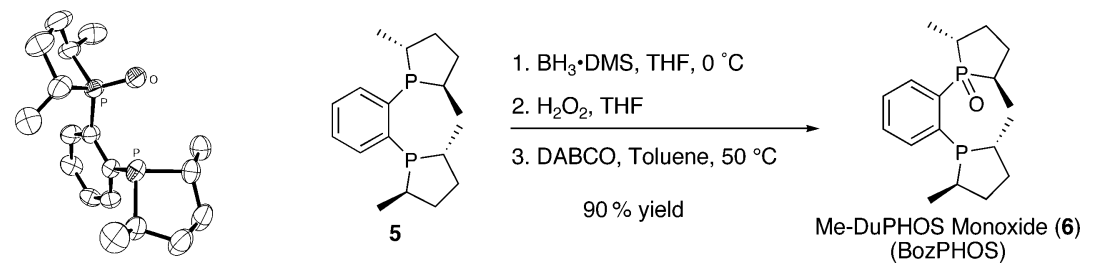

Scheme 1

The reactivity and selectivity of this novel ligand was far superior to those observed with Me-DuPHOS. This was demonstrated by two reactions involving the addition of dimethylzinc to the $N$-phosphinoylimine derived from benzaldehyde, one catalyzed by Me-DuPHOS $\cdot \mathrm{Cu}$ and the other by BozPHOS $\cdot \mathrm{Cu}$ (eqs. 6-7). The yield went up to $93 \%$ with only 3 equiv of dimethylzinc and the ee to $97 \%$ when BozPHOS $\cdot \mathrm{Cu}$ was used.<smiles>O=P(c1ccccc1)(c1ccccc1)c1ccccc1</smiles>
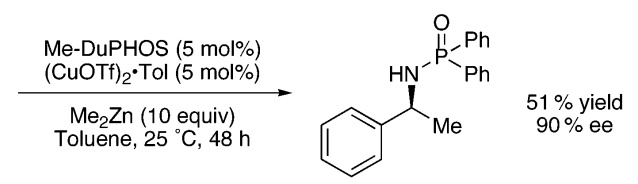<smiles>O=P(c1ccccc1)(c1ccccc1)c1ccccc1</smiles>
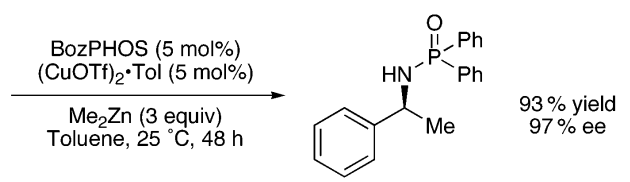

These studies led to a second-generation chiral ligand, Me-DuPHOS monoxide (BozPHOS), which not only significantly improved the enantioselectivities of the process, but also led to a broader scope in terms of the diorganozinc reagents used in the reaction (Table 1) [14]. The addition reaction was very effective with a wide range of imines derived from arylaldehydes. 
Table 1 Addition of dialkylzinc to $N$-phosphinoylimines catalyzed by BozPHOS/Cu(OTf $)_{2}$.

$\mathrm{Cu}(\mathrm{OTf})_{2}(6 \mathrm{~mol} \%)$
$\mathrm{BozPHOS}(6)(3 \mathrm{~mol} \%)$

The preparation of imines derived from alkylaldehydes was much more problematic. Although it was possible to prepare $N$-diphenylphosphinoylimines derived from alkylaldehydes bearing enolizable protons in ca. 50-60 \% yields using Stec's procedure [15,16], the product was not sufficiently clean to be used in the copper-catalyzed reaction. Furthermore, it could not be purified using standard methods without extensive decomposition. Therefore, we elected to use an imine precursor [17-19] in which the leaving group would depart upon treatment with the dialkylzinc reagent (Fig. 2). Obviously, the nature of the by-product (RZnLG) should not interfere with the asymmetric catalytic process. Among those that were surveyed, the sulfinate adduct turned out to be the best one.

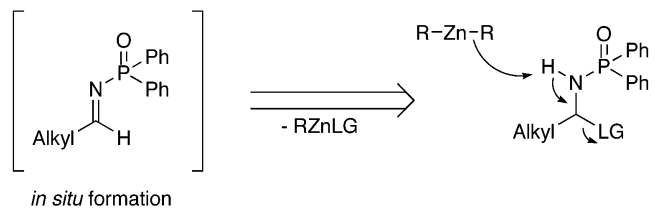

Fig. 2

Finally, the scope of the reaction was significantly increased when we found that the sulfinic acid adduct of the starting $N$-phosphinoylimine was an excellent surrogate for $N$-phosphinoylimines derived from alkyl-substituted aldehydes. The starting materials were simply prepared by mixing the aldehyde, $p$-toluenesulfinic acid, and diphenylphosphinic amide in an etheral solvent (ether or $t$-butyl methyl ether). After stirring for several hours, the adduct precipitated and could be isolated by filtration. Submission of the adduct to the standard conditions but using an extra equivalent of the dialkylzinc led to the addition products in high yields and enantiomeric excesses (Table 2) [20]. This method is among the best, if not the best, to generate this class of compounds. 
Table 2 Addition of dialkylzinc to $N$-phosphinoylalkylimines.

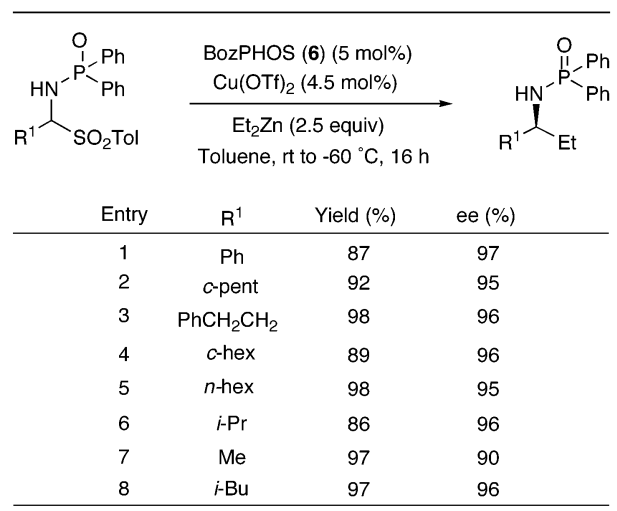

A careful analysis of the ligand indicates that Me-DuPHOS monoxide is the optimal one thus far. Replacement of the methyl groups with ethyl or isopropyl led to a significant erosion of the enantioselectivity and reaction rate (Fig. 3). Furthermore, we have also demonstrated that the presence of both, the phospholane and phosphine oxide, is mandatory to get high enantioselectivities. If either one of the groups is removed, the yield is still very good, but no enantioselectivity was observed.

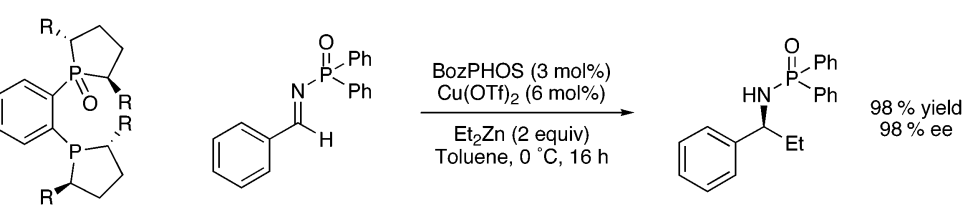
BozPHOS $(\mathrm{R}=\mathrm{Me})$

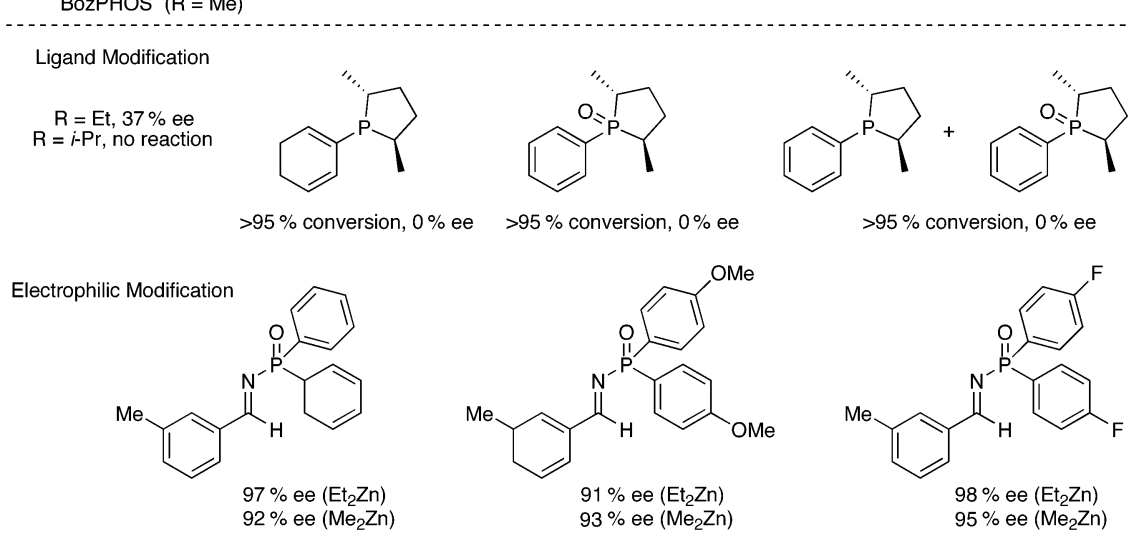

Fig. 3

Finally, changing the electronic nature of the aryl group of the diarylphosphinoylimine is translated into a slight modification of the enantioselectivities. We observed the presence of a 4-fluoro substituent increases the ee in some cases, whereas the presence of a 4-methoxy led to a decrease in ee and reaction rates (Fig. 3).

The next important question that needed to be addressed was the possibility that both the Me-DuPHOS $\cdot \mathrm{Cu}$ catalyzed reaction and the BozPHOS $\cdot \mathrm{Cu}$ catalyzed reaction were proceeding via the same active catalytic species. In other words, could it be possible that under the reaction conditions, one of the copper salts could oxidize Me-DuPHOS into BozPHOS (eq. 8)? Such phosphine oxidation is well 
precedented with palladium chemistry [12c,21], and it has also been reported for copper sulfate and dppe [22].

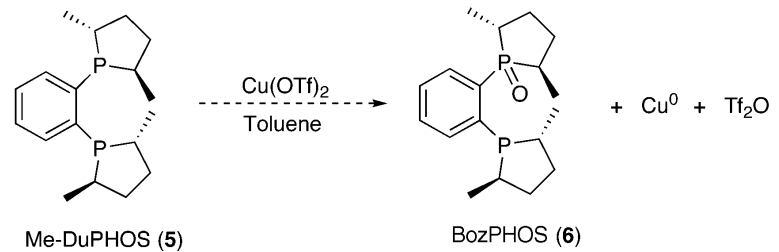

In order to establish this, we developed two different protocols (Fig. 4). A high level of enantioselectivities was observed with both ligands if $\mathrm{Cu}(\mathrm{OTf})_{2}$ was mixed with Me-DuPHOS or BozPHOS prior to the addition of $\mathrm{Et}_{2} \mathrm{Zn}$. Quite surprisingly, the racemic $N$-phosphinoyl protected amine was observed with Me-DuPHOS if the $\mathrm{Cu}$ (II) salt was initially reduced with $\mathrm{Et}_{2} \mathrm{Zn}$ (presumably to generate $\mathrm{EtCu}$ ) followed by the addition of the chiral ligand. Conversely, the order of addition did not have any impact on the ee of the product if BozPHOS was used.

\begin{tabular}{|c|c|c|}
\hline & Original Procedure with Me-DuPHOS & "Non-oxidizing" Procedure \\
\hline & $\begin{array}{l}\text { 1. } \mathrm{Cu}(\mathrm{OTf})_{2}+\text { Ligand + Toluene } \\
\text { 2. Add } \mathrm{Et}_{2} \mathrm{Zn} \\
\text { 3. Add Imine }\end{array}$ & $\begin{array}{l}\text { 1. } \mathrm{Cu}(\mathrm{OTf})_{2}+\mathrm{Et}_{2} \mathrm{Zn}+\text { Toluene } \\
\text { 2. Add Ligand } \\
\text { 3. Add Imine }\end{array}$ \\
\hline Me-DuPHOS (5) & $\begin{array}{c}94 \% \\
96 \% \mathrm{ee}\end{array}$ & $\begin{array}{l}45 \% \\
2 \% \text { ee }\end{array}$ \\
\hline BozPHOS (6) & $\begin{array}{c}98 \% \\
98 \% \text { ee }\end{array}$ & $\begin{array}{c}98 \% \\
98 \% \text { ee }\end{array}$ \\
\hline Me-DuPHOS bisoxide (7) & $\begin{array}{l}50 \% \text { conversion } \\
0 \% \text { ee }\end{array}$ & $\begin{array}{l}50 \% \text { conversion } \\
0 \% \text { ee }\end{array}$ \\
\hline
\end{tabular}

Fig. 4

To demonstrate whether Me-DuPHOS oxidation to generate BozPHOS in situ was a viable pathway under the normal addition protocol (ligand + copper salt, then $\mathrm{Et}_{2} \mathrm{Zn}$ ), we undertook a systematic spectroscopic investigation of the reaction to identify whether or not, BozPHOS (6) or Me-DuPHOS bisoxide (7) were formed upon treatment with various $\mathrm{Cu}$ salts. Unfortunately, the in situ monitoring of the reaction by NMR methods did not lead to unambiguous results owing to the presence of paramagnetic $\mathrm{Cu}(\mathrm{II}) / \mathrm{Cu}(0)$ species and the rapid equilibration between various complexes.

To overcome this problem, we had to scavenge all the residual $\mathrm{Cu}$ salts from the crude reaction mixture by an aqueous KCN treatment under deoxygenated conditions. This is a convenient alternative to the use of dithiocatechol dilithio salts that has been used to scavenge and recover phosphine ligands [23]. Each ligand (5-7) was submitted to $\mathrm{Cu}(\mathrm{OTf})_{2} / \mathrm{Et}_{2} \mathrm{Zn}$ using the normal or reverse addition protocol. After a standard KCN work-up to remove copper salts, the crude mixture was analyzed by ${ }^{31} \mathrm{P}$ NMR. The first striking observation is that significant oxidation (up to $20 \%$ ) of one of the free phosphino groups of Me-DuPHOS occurred when the ligand was premixed with $\mathrm{Cu}(\mathrm{OTf})_{2}$ (eq. 9). Conversely, inverting the order of addition almost completely suppressed the oxidation of the phosphine (only $3 \%$ of BozPHOS was formed, eq. 10). The same series of experiments carried with BozPHOS (6) indicated that very little oxidation into 7 (1-4\%) was observed regardless of the order of addition. This is somewhat not too surprising since the monoxide should be less prone to oxidation compared to Me-DuPHOS due to the replacement of a phosphine by a phosphine oxide. The relatively low level of oxidation using the nonoxidizing procedure could potentially be attributed to the background oxidation during the manipulations under a noninert atmosphere and not to a formal oxidation of phosphorus by the in situ formed $\mathrm{EtCu}$. 


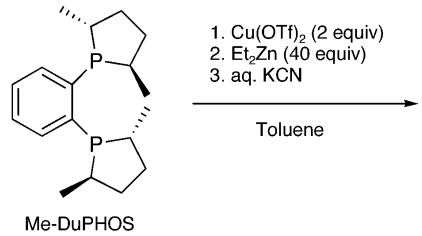

\begin{tabular}{l} 
1. $\mathrm{Et}_{2} \mathrm{Zn}(40$ equiv) \\
2. $\mathrm{Cu}(\mathrm{OTf})_{2}$ ( 2 equiv) \\
3. Ligand (1 equiv) \\
4. aq. $\mathrm{KCN}$ \\
\multicolumn{1}{c}{ Toluene }
\end{tabular}

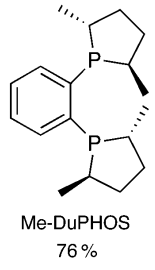

$96 \%$
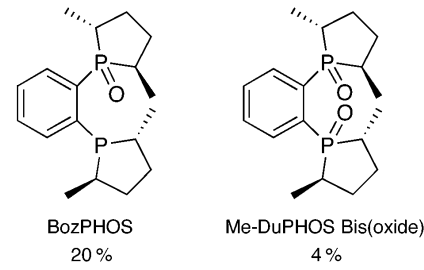

$4 \%$

$1 \%$

Kinetic studies of the reaction as a function of the ligand using the reverse addition protocol are shown in Fig. 5. The data strongly suggest that the ligand 6 is responsible for the highly enantioselective pathway since the reaction involving $\mathrm{Cu}(\mathrm{I}) \cdot \mathbf{5}$ under nonoxidizing condition led to a low rate and poor enantioselectivities. One striking feature is the reactivity difference between the complex $\mathrm{Cu}(\mathrm{I}) \cdot \mathbf{5}$ and $\mathrm{Cu}(\mathrm{I}) \cdot 6$ in the reaction, and it accounts for the observation that high enantioselectivities are observed for the reaction even when a small amount of $\mathrm{Cu}(\mathrm{I}) \cdot \mathbf{6}$ was present relative to $\mathrm{Cu}(\mathrm{I}) \cdot \mathbf{5}$.

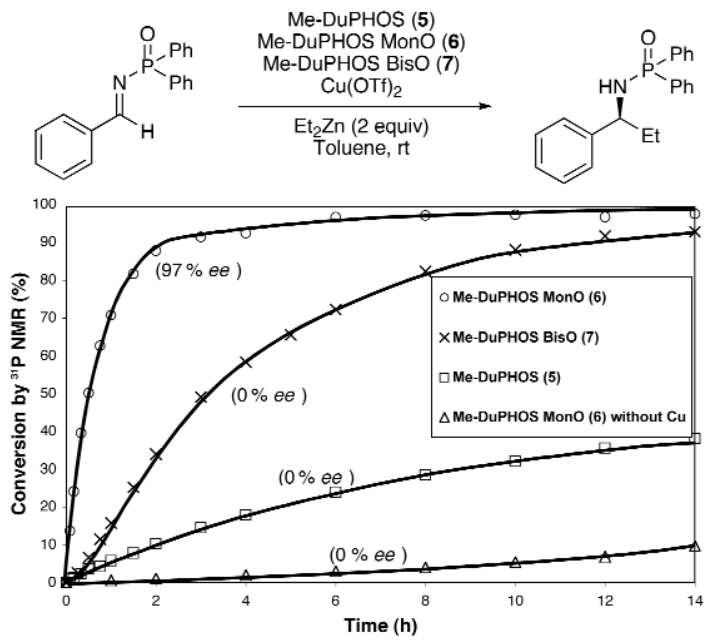

Fig. 5

In conclusion, these findings are potentially very important owing to the large number of reports involving phosphine-bound copper catalysts in asymmetric catalysis. In a lot of cases, a careful examination that the potentially active chiral ligand could be the corresponding phosphine oxide should be carried out and enantioselectivities may be improved in many cases.

\section{ACKNOWLEDGMENTS}

This work was supported by NSERC, Merck Frosst Canada, Boehringer Ingelheim (Canada), AstraZeneca, and the Université de Montréal. A. A. B. and C. L. are grateful to NSERC (PGF B) and F. C. A. R. (B2) for postgraduate fellowships. A. C. is grateful to NSERC (ES). J.-N. D. is grateful to NSERC (ES) and FQRNT (B1). 


\section{REFERENCES AND NOTES}

1. Reviews: (a) R. Bloch. Chem. Rev. 98, 1407-1438 (1998); (b) S. Kobayashi and H. Ishitani. Chem. Rev. 99, 1069-1094 (1999).

2. For a review of earlier reports, see: S. E. Denmark and O. J. C. Nicaise. In Comprehensive Asymmetric Catalysis, Vol. II, E. N. Jacobsen, A. Pfaltz, H. Yamamoto (Eds.), pp. 921-961, Springer, Berlin (1999) and refs. cited therein.

3. (a) H. Fujihara, K. Nagai, K. Tomioka. J. Am. Chem. Soc. 122, 12055-12056 (2000); (b) T. Soeta, K. Nagai, H. Fujihara, M. Kuriyama, K. Tomioka. J. Org. Chem. 68, $9723-9727$ (2003).

4. (a) J. R. Porter, J. F. Traverse, A. H. Hoveyda, M. L. Snapper. J. Am. Chem. Soc. 123, 984-985 (2001); (b) J. R. Porter, J. F. Traverse, A. H. Hoveyda, M. L. Snapper. J. Am. Chem. Soc. 123, 10409-10410 (2001).

5. (a) S. Dahmen and S. Bräse. J. Am. Chem. Soc. 124, 5940-5941 (2002); (b) N. Hermanns, S. Dahem, C. Bolm, S. Bräse. Angew. Chem., Int. Ed. 41, 3692-3694 (2002).

6. C.-J. Wang and M. Shi. J. Org. Chem. 68, 6229-6237 (2003).

7. X. Li, L.-F. Cun, L.-Z. Gong, A.-Q. Mi, Y.-Z. Jiang. Tetrahedron: Asymmetry 14, 3819-3821 (2003).

8. For the use of $N$-phosphinoylimines, see: (a) S. Masumoto, H. Usuda, M. Suzuki, M. Kanai, M. Shibasaki. J. Am. Chem. Soc. 125, 5634-5635 (2003); (b) S. Matsunaga, N. Kumagai, S. Harada, M. Shibasaki. J. Am. Chem. Soc. 125, 4712-4713 (2003); (c) S. Matsunaga, T. Yoshida, H. Morimoto, N. Kumagai, M. Shibasaki. J. Am. Chem. Soc. 126, 8777-8785 (2004); (d) P. Wipf, C. Kendall, C. R. J. Stephenson. J. Am. Chem. Soc. 125, 761-768 (2003); (e) P. Wipf and C. R. J. Stephenson. Org. Lett. 5, 2449-2452 (2003); (f) B. H. Lipshutz and H. Shimizu. Angew. Chem., Int. Ed. 43, 2228-2230 (2004); (g) K.-I. Yamada, S. J. Harwood, H. Gröger, M. Shibasaki. Angew. Chem., Int. Ed. 38, 3504-3506 (1999); (i) T. Okino, S. Nakamura, T. Furukawa, Y. Takemoto. Org. Lett. 6, 625-627 (2004).

9. Calculations of the LUMO energy [geometry optimized at B3LYP/6-31G(d)] indicates the following relative energy $(\mathrm{eV})$ of various imines derived from benzaldehyde relative to the $N$-methylmine: $N$-methyl imine (0), $N$-phenyl (-0.49), benzaldehyde $(-0.63), N$-diphenylphosphinoyl $(-0.65), N$-Ts $(-0.95), N$-benzoyl $(-1.25)$.

10. (a) C. Jimeno, K. S. Reddy, L. Sola, A. Moyano, M. A. Pericas, A. Riera. Org. Lett. 2, 3157-3159 (2000); (b) I. Sato, R. Kodaka, K. Soai. J. Chem. Soc., Perkin Trans. 1 2912-2914 (2001); (c) P. Pinho and P. G. Andersson. Tetrahedron 57, 1615-1618 (2001); (d) H. L. Zhang, X. M. Zhang, L. Z. Gong, A. Q. Mi, X. Cui, Y. Z. Jiang, M. C. K. Choi, A. S. C. Chan. Org. Lett. 4, 1399-1402 (2002); (e) X.-M. Zhang, H.-L. Zhang, W.-Q. Lin, L.-Z. Gong, A.-Q. Mi, X. Cui, Y.-Z. Jiang, K.-B. Yu. J. Org. Chem. 68, 4322-4329 (2003); (f) D. Guijarro, P. Pinho, P. G. Anderson. J. Org. Chem. 63, 2530-2535 (1998); (g) X. Zhang, L. Gong, A. Mi, X. Cui, Y. Jiang, M. C. K. Choi, A. S. C. Chan. Tetrahedron Lett. 42, 6369-6372 (2001); (h) K. J. M. Beresford. Tetrahedron Lett. 43, 7175-7177 (2002); (i) P. G. Anderson, D. Guijarro, D. Tanner. J. Org. Chem. 62, 7364-7375 (1997).

11. A. A. Boezio and A. B. Charette. J. Am. Chem. Soc. 125, 1692-1693 (2003).

12. Only a few chiral BPMO ligands have been reported thus far: (a) W. J. Marshall and V. V. Grushin. Organometallics 22, 555-562 (2003); (b) V. V. Grushin. Organometallics 20, 3950-3961 (2001); (c) V. V. Grushin. J. Am. Chem. Soc. 121, 5831-5832 (1999); (d) R. J. Coye, Y. L. Slovokhotov, M. Y. Antipin, V. V. Grushin. Polyhedron 17, 3059-3070 (1998).

13. V. V. Grushin. Chem. Rev. 104, 1629-1662 (2004).

14. A. A. Boezio, J. Pytkowicz, A. Côté, A. B. Charette. J. Am. Chem. Soc. 125, 14260-14261 (2003).

15. B. Krzyzanowska and W. J. Stec. Synthesis 521-524 (1978).

16. L. A. Arini, A. Sinclair, P. Szeto, R. A. Stockman. Tetrahedron Lett. 45, 1589-1591 (2004). 
17. A. R. Katritzky and P. A. Harris. Tetrahedron: Asymmetry 3, 437-442 (1992).

18. T. Mecozzi and M. Petrini. J. Org. Chem. 64, 8970-8972 (1999).

19. H. Kohn, K. A. Sawhney, D. W. Robertson, J. D. Leander. J. Pharm. Sci. 83, 689-691 (1994).

20. A. Côté, A. A. Boezio, A. B. Charette. Proc. Natl. Acad. Sci. USA 101, 5405-5410 (2004).

21. (a) F. Ozawa, A. Kubo, Y. Matsumoto, T. Hayashi, E. Nishioka, K. Yanagi, K. Moriguchi. Organometallics 12, 4188-4196 (1993); (b) C. Amatore, E. Carré, A. Jutand, M. A. M'Barki. Organometallics 14, 1818-1826 (1995).

22. S. J. Berners-Price, R. K. Johnson, C. K. Mirabelli, L. F. Faucette, F. L. McCabe, P. Sadler. J. Inorg. Chem. 26, 3383-3387 (1987).

23. B. H. Lipshutz, B. Frieman, H. Birkedal. Org. Lett. 6, 2305-2308 (2004). 